\section{Avaliação da anestesia epidural com lidocaína associada ao tramadol ou à dexmedetomidina, em gatas submetidas à ovariosalpingohisterectomia, anestesiadas com propofol}

[Evaluation of epidural anesthesia with lidocaine associated to tramadol or dexmedetomidine, in cats undergoing ovariosalpingohisterectomy, anesthetized with propofol]

J.Q. Sande ${ }^{1}$, T.B.S. Souza ${ }^{2}$, I.L.G. Kersul ${ }^{1}$, N.P. Anjos ${ }^{3}$, E.F. Martins Filho ${ }^{4}$, V.F. Barbosa ${ }^{4}$

${ }^{1}$ Residência - Universidade Federal da Bahia - Salvador, BA ${ }^{2}$ Programa de pós-graduação - Universidade Federal da Bahia - Salvador, BA ${ }^{3}$ Programa de graduação - Universidade Federal da Bahia - Salvador, BA ${ }^{4}$ Universidade Federal da Bahia - Salvador, BA

\title{
RESUMO
}

Objetivou-se comparar os efeitos fisiológicos, analgésicos e sobre a taxa de infusão de propofol, decorrentes da anestesia epidural com lidocaína, associada ao tramadol ou à dexmedetomidina, em felinas submetidas à ovariosalpingohisterectomia $(\mathrm{OSH})$. Para tal, 16 felinas hígidas foram pré-tratadas com acepromazina 0,08mg/kg/IM, utilizando-se propofol para a indução (dose-efeito) e manutenção anestésicas. Após indução, as gatas foram aleatoriamente distribuídas em dois grupos $(n=8)$, designados: grupo lidocaína-tramadol (GLT), tratado com lidocaína $(3,0 \mathrm{mg} / \mathrm{kg})$ associada ao tramadol $(2,0 \mathrm{mg} / \mathrm{kg})$; e grupo lidocaína-dexmedetomidina $(\mathrm{GLD})$, tratado com lidocaína $(3,0 \mathrm{mg} / \mathrm{kg})$ associada à dexmedetomidina $(2 \mu \mathrm{g} / \mathrm{kg})$, pela via epidural. Durante a OSH, a infusão de propofol foi aumentada ou reduzida, objetivando-se manutenção de plano anestésico cirúrgico. Foram avaliados os parâmetros: $f, \mathrm{FC}, \mathrm{SPO}_{2}, \mathrm{EtCO}_{2}, \mathrm{PAS}, \mathrm{PAD}, \mathrm{PAM}, \mathrm{T}^{\circ} \mathrm{C}$, nos períodos pré (M1) e transoperatórios (M2 a M7); a taxa mínima de propofol necessária; o tempo de recuperação anestésica e a qualidade da analgesia pós-cirúrgica durante seis horas. Ambos os tratamentos garantiram baixas taxas mínimas de infusão de propofol, todavia o uso da dexmedetomidina resultou em bradicardia inicial, elevação da pressão arterial, maior tempo de recuperação e menor qualidade analgésica, quando comparada ao tramadol.

Palavras-chave: alfa-2 agonista, opioide, anestesia locorregional

\begin{abstract}
The aim of this study was to compare the physiological and analgesic effects and the minimum infusion rate of propofol of epidural anesthesia with lidocaine associated to tramadol or dexmedetomidine, in cats undergoing ovariosalpingohysterectomy (OSH). For this purpose, 16 healthy cats were pretreated with acepromazine $\left(0.08 \mathrm{mg} \mathrm{kg}^{-1} \mathrm{IM}\right)$ and propofol was used for induction (dose-effect) and maintenance of anesthesia. After induction, the cats were assigned in two randomized groups $(n=8)$, named: Lidocaine-tramadol group $(L T G)$, treated with lidocaine $\left(3 \mathrm{mg} \mathrm{kg}^{-1}\right)$ associated to tramadol $\left(2 \mathrm{mg} \mathrm{kg}^{-1}\right)$ and Lidocaine-dexmedetomidine group $(L D G)$, treated with lidocaine $\left(3 \mathrm{mg} \mathrm{kg}^{-1}\right)$ associated to dexmedetomidine $\left(2 \square \mathrm{g} \mathrm{kg}^{-1}\right)$, by epidural route. During OSH, propofol infusion was increased or decreased, setting to maintain surgical anesthetic depth. The parameters f, $\mathrm{HR}, \mathrm{SPO}_{2}, \mathrm{EtCO}_{2}, \mathrm{SAP}, \mathrm{DAP}, \mathrm{MAP}, \mathrm{T}^{\circ} \mathrm{C}$ in the pre (M1) and trans-operative periods (M2 to M7); minimum infusion rate of propofol; time of anesthetic recovery and quality of postoperative analgesia during six-hour interval, were evaluated. Both protocols provided low minimum infusion rate of propofol. However, dexmedetomidine resulted in initial bradycardia, elevated blood pressure, longer recovery time, and lower analgesic quality when compared to tramadol.
\end{abstract}

Keywords: alpha-2 agonist, opioid, locoregional anesthesia

Recebido em 18 de janeiro de 2018

Aceito em 26 de março de 2019

E-mail: jessica.sande92@gmail.com 


\section{INTRODUÇÃO}

A ovariosalpingohisterectomia (OSH) em felinos apresenta-se como procedimento cirúrgico corriqueiro na prática veterinária e de extrema importância para controle populacional e prevenção de patologias hormônio-dependentes. É vinculada à dor de intensidade moderada, consistindo em modelo cirúrgico para estudos de protocolos anestésicos e analgésicos, com o objetivo de controle eficiente e seguro da dor aguda (Pohl et al., 2012).

$\mathrm{Na}$ expectativa da prevenção e do tratamento da dor durante os períodos trans e pós-operatórios, a anestesia epidural mostra-se uma alternativa, capaz de promover anestesia e analgesia adequadas, sobretudo ao se associar anestésicos locais e analgésicos de diferentes classes farmacológicas. Tais combinações extradurais potencializam anestésicos intravenosos e inalatórios, com redução de efeitos depressores e maior estabilidade do plano anestésico (Sonaglio et al., 2014). Dentre os anestésicos locais, a lidocaína destaca-se em razão da curta latência, alta lipossolubilidade, toxicidade moderada e duração de ação de aproximadamente 60 a 120 minutos, quando associada à epinefrina (Torske e Dyson, 2000; DeRossi et al., 2009). O cloridrato de tramadol apresenta-se como fármaco opioide hidrossolúvel, característica que lhe confere ação epidural prolongada e progressão cranial, quando associado aos anestésicos locais. Produz analgesia moderada e supressão da maioria dos efeitos adversos dos opioides, porém com ação espinhal semelhante (Sonaglio et al., 2014; Hermeto et al., 2015).

A dexmedetomidina, classificada como $\alpha-2$ agonista adrenérgico altamente específico, diminui a liberação central e periférica de noradrenalina, proporcionando efeitos sedativos e analgésicos. Pela via epidural, promove analgesia por interação direta com receptores espinhais, sem instaurar bloqueio motor. Seus efeitos cardiovasculares são dose-dependentes, contudo não promove depressão respiratória significativa (Silva et al., 2008; Dorigon et al., 2009; Caires e Clark, 2014; Sonaglio et al., 2014).

Apesar da amplitude de estudos envolvendo associações analgésicas e anestésicas, a literatura é escassa no que se refere a protocolos eficazes à espécie felina, sobretudo no que concerne à via extradural. Dessa forma, objetivou-se avaliar os efeitos peridurais do tramadol e da dexmedetomidina, quando associados à lidocaína, sobre a taxa de infusão de propofol, as variáveis fisiológicas, o tempo de recuperação anestésica e a analgesia pós-cirúrgica, em gatas submetidas à $\mathrm{OSH}$.

\section{MATERIAL E MÉTODOS}

O presente estudo foi aprovado pelo Comitê de Ética da Escola de Medicina Veterinária e Zootecnia da Universidade Federal da Bahia (EMEVZ-UFBA), sob protocolo de número $50 / 2017$.

Foram utilizadas 16 gatas de raças variadas, adultas, consideradas hígidas após avaliação clínica e laboratorial (hemograma e perfis enzimáticos renal e hepático). As fêmeas foram recebidas 24 horas prévias ao protocolo experimental e acondicionadas em gatis para adaptação ambiental, estabelecendo-se, sequencialmente, jejum alimentar e hídrico de 12 e duas horas, respectivamente. A seguir, foram distribuídas aleatoriamente em dois grupos de oito animais cada, denominados: grupo lidocaína-tramadol (GLT) e grupo lidocaínadexmedetomidina (GLD).

Após medicação pré-anestésica (MPA) com $0,08 \mathrm{mg} / \mathrm{kg}$ IM de acepromazina $0,2 \%$ (Apromazin $^{\circledR}$, maleato de acepromazina, Syntec, Brasil), procedeu-se à cateterização das veias cefálicas bilaterais, instituindo-se fluidoterapia com solução de ringer lactato $(3 \mathrm{~mL} / \mathrm{kg} / \mathrm{h})$ e a administração de cefalotina sódica (cefalotina sódica, genérico, ABL, Brasil) (30mg/kg IV) e de meloxicam Maxicam $^{\circledR} 0,2 \%$, Ourofino, Brasil) $(0,1 \mathrm{mg} / \mathrm{kg}$ IV), além de tricotomia das regiões abdominal e sobre o dorso do paciente, abrangendo-se a área sobre o espaço vertebral lombossacral e seu entorno. Decorridos 20 minutos da MPA, os animais foram induzidos à anestesia com propofol (Propovan ${ }^{\circledR}$, propofol, Cristália, Brasil), em dose suficiente para intubação orotraqueal a uma taxa de $6 \mathrm{mg} / \mathrm{kg} / \mathrm{min}$, administrada por meio de bomba de infusão (Infusion Pump 680T ${ }^{\circledR}$, Samtronic Infusion Systems, Brasil), seguindo-se a manutenção com o mesmo fármaco $(0,3 \mathrm{mg} / \mathrm{kg} / \mathrm{min})$. As fêmeas permaneceram sob 
ventilação espontânea com oxigênio a $100 \%$ $\left(\mathrm{FiO}_{2}=1,0\right)$, em circuito sem reinalação de gases.

Ato contínuo, foram posicionadas em decúbito esternal sobre colchão térmico ativo e, com os membros pélvicos flexionados cranialmente, receberam antissepsia com solução alcoólica de clorexidina a $0,2 \%$ sobre o espaço epidural lombossacro (L7-S1), o qual foi acessado com agulha de Tuohy 22G (Agulha Peridural BD Tuohy ${ }^{\circledR}, \quad$ BD, Brasil), confirmando-se a localização pelo teste da gota pendente ou pela perda da resistência à aplicação de ar $(0,2 \mathrm{~mL})$. A seguir, por essa via, o grupo GLT recebeu $3,0 \mathrm{mg} / \mathrm{kg}$ de lidocaína $2 \%$ (Xylestesin $^{\circledR}$, cloridrato de lidocaína, Cristália, Brasil) associada a $2,0 \mathrm{mg} / \mathrm{kg}$ de cloridrato de tramadol (Tramadon $^{\circledR}$, cloridrato de tramadol, Cristália, Brasil) e, no grupo GLD, administrou-se $3,0 \mathrm{mg} / \mathrm{kg}$ de lidocaína associado a $2 \mu \mathrm{g} / \mathrm{kg}$ de dexmedetomidina (Dexdomitor ${ }^{\circledR}$, cloridrato de dexmedetomidina, Zoetis, Brasil). O volume de solução foi complementado com $\mathrm{NaCl} 0,9 \%$, totalizando-se $0,3 \mathrm{~mL} / \mathrm{kg}$, injetado em um período de 30 segundos.

Decorridos 10 minutos da aplicação epidural, as gatas foram posicionadas em decúbito dorsal, procedendo-se à antissepsia da região retroumbilical. A OSH, por incisão na linha alba, foi executada por equipe cirúrgica única, estipulando-se um tempo operatório médio de 45 minutos. No decorrer do ato cirúrgico, a taxa de propofol foi alterada, objetivando-se a manutenção de oferta anestésica suficiente para inibir resposta ao estímulo álgico, considerandose, mediante as características oculares, o plano: superficial, com o globo ocular centralizado e presença de reflexos palpebrais; adequado, com o globo ocular rotacionado, ausência de reflexo palpebral lateral e ausência ou presença diminuída de reflexo palpebral medial ou profundo, com o globo ocular centralizado e ausência de reflexos palpebrais (Comassetto et al., 2015).

O momento inicial de avaliação (M1) foi estipulado 10 minutos após a indução anestésica, ajustando-se a taxa de infusão mínima de propofol nos momentos de estímulo nociceptivo supramáximo considerando-se: M2 (final da celiotomia); M3 (após pinçamento do ovário direito); M4 (após pinçamento do ovário esquerdo); M5 (após pinçamento da cérvix uterina); M6 (após sutura muscular abdominal) e M7 (após sutura de pele). Nesses momentos, quando houve diminuição maior que $20 \%$, em relação ao M1, das variáveis frequência cardíaca (FC), frequência respiratória $(f)$ e pressão arterial média (PAM), associada às características oculares de plano profundo, a taxa de infusão de propofol foi diminuída em $0,05 \mathrm{mg} / \mathrm{kg} / \mathrm{min}$. Quando, no entanto, a analgesia foi considerada insuficiente, evidenciada pelo aumento de $20 \%$ em relação ao M1 dessas mesmas variáveis, associado às características oculares de plano superficial, a taxa de infusão de propofol foi acrescida de $0,05 \mathrm{mg} / \mathrm{kg} / \mathrm{min}$. Diante da estabilidade das variáveis cardiorrespiratórias com evidência de plano anestésico adequado, manteve-se a taxa de propofol, suspendendo-se sua administração ao término do procedimento cirúrgico. A determinação da taxa média de infusão do propofol, para cada momento, nos diferentes grupos, foi calculada com base no consumo total do fármaco, considerando-se $\mathrm{o}$ peso dos animais e o tempo de infusão. A taxa média final de propofol correspondente a cada grupo foi calculada como a média aritmética das taxas de infusão dos diferentes momentos de avaliação.

Foram monitorados e registrados os dados de FC, $f$, saturação da oxihemoglobina $\left(\mathrm{SpO}_{2}\right)$, tensão do dióxido de carbono ao final da expiração $\left(\mathrm{EtCO}_{2}\right)$, pressão arterial sistólica (PAS), pressão arterial diastólica (PAD), PAM e temperatura retal $\left(\mathrm{T}^{\circ} \mathrm{C}\right)$ por leitura direta em monitor multiparamétrico veterinário (LifeWindowLW9xVet ${ }^{\circledR}$, Digicare Biomedical Technology Inc, Florida, USA), nos momentos anteriormente descritos (M1 a M7).

Após a extubação, a avaliação analgésica pósoperatória foi realizada por observadores treinados, utilizando-se a escala multidimensional para avaliação da dor aguda pós-operatória em gatos (Brondani et al., 2012), determinando-se sequencialmente um escore final, que, quando igual ou superior a oito, implicou na administração de analgesia complementar com cloridrato de tramadol $(2 \mathrm{mg} / \mathrm{kg}$ IV). Os registros dos parâmetros pertinentes à escala iniciaram-se 30 minutos após o término do procedimento cirúrgico (M30), com novos registros em intervalos de 30 minutos (M60, M90, M120). A partir do M120, as avaliações foram registradas a cada hora (M180, 
M240 e M360). Adicionalmente, foram notificadas as ocorrências de vômitos, excitação e edema pulmonar, bem como a duração da recuperação anestésica, considerada como o tempo entre a extubação e a primeira tentativa de levantamento da cabeça.

Para a avaliação entre momentos dentro de cada grupo, foi utilizado o teste de Friedman, para as variáveis qualitativas, e a análise de variância de uma via para medidas repetidas (One way ANOVA repeated meansures) para as variáveis quantitativas. Para a avaliação entre grupos dentro de cada momento, foi usado o teste $t$ de Student para amostras independentes, para parâmetros quantitativos, e o teste WilcoxonMann-Whitney, para as variáveis qualitativas. $\mathrm{O}$ teste de normalidade utilizado foi o ShapiroWilk. O nível de significância adotado foi de $5 \%$.

\section{RESULTADOS E DISCUSSÃO}

Cabe mencionar que, na expectativa da obtenção de anestesia abrangente e adequada à técnica de $\mathrm{OSH}$, optou-se por utilizar um volume epidural final de $0,3 \mathrm{~mL} / \mathrm{kg}$. A literatura refere como rotineira a punção lombossacra (L7-S1), atingindo-se, em felinos, bloqueio da região ao nível de L2-L1 $(0,2 \mathrm{~mL} / \mathrm{kg})$ e dermátomos até T7 $(0,3 \mathrm{~mL} / \mathrm{kg})$ (Valverde, 2008; Otero, 2013). Lee et al. (2004) corroboram a assertiva ao atestarem progressão entre $\mathrm{T} 7$ e $\mathrm{T} 11$ relativa à administração de $0,3 \mathrm{~mL} / \mathrm{kg}$ de azul de metileno no espaço epidural (L7-S1) de felinos. Em estudo recente, o uso epidural (L7-S1) de lidocaína associada à morfina ou à metadona em volume final de $0,25 \mathrm{~mL} / \mathrm{kg}$, determinou analgesia adequada e prolongada em felinas submetidas à OSH (DeRossi et al., 2016). Complementarmente, a associação tramadollidocaína, em doses idênticas à do estudo em pauta, demonstrou analgesia superior ao uso do anestésico local isolado, com propagação cranial satisfatória (T13-L1) em felinas (Hermeto et al., 2015).

Relativamente a variáveis cardiorrespiratórias (Tab. 1), constatou-se menor FC no grupo GLD em relação ao GLT no M2 ( $\mathrm{P}=0,019)$, o que pode ser imputado à ação da dexmedetomidina, que, apesar de altamente seletiva, preserva efeitos cronotrópicos negativos típicos dos alfa-2 agosnistas (Souza et al., 2010; Pohl et al., 2012; Caires e Clark, 2014; Rodrigo-Mocholi et al., 2016; Kim et al., 2017). A comparação da dexmedetomidina com o tramadol, associados ao midazolam em felinas, resultou em efeito semelhante da primeira, embora administrados IM (Lemos et al., 2017). Contudo, vale salientar que não foram constadas alterações de ritmo cardíaco, como bloqueios atrioventriculares, diante da associação epidural com lidocaína, detectando-se, sequencialmente, médias constantes nos limites de normalidade (Camacho e Mucha, 2014). Em adição, cabe mencionar a diminuição dos valores iniciais da FC no M2, para ambos os tratamentos, possivelmente em razão da estabilidade anestésica conferida pela técnica epidural, em combinação ao efeito simpatolítico do propofol extensivamente reportado na literatura (Oliveira et al., 2007).

A avaliação das pressões arteriais denotou menores médias nos animais tratados com tramadol em relação à dexmedetomidina, em especial nos momentos pós-celiotomia (M2) e após a ligadura da cérvix (M5). Portanto, apesar dos reconhecidos efeitos vasodilatadores da acepromazina e do propofol (Koch et al., 2008; Rankin, 2015) associados àqueles da administração epidural de lidocaína (Kvolik e Sakic, 2007), pode-se notar que a dexmedetomidina incrementou a resistência vascular periférica em razão de sua atividade adrenérgica primária decorrente de seu típico efeito bifásico (Kim et al., 2017) até o momento da celiorrafia. Outros autores reportam efeito vasoconstrictor similar com o uso da dexmedetomidina isolada ou associada a agentes anestésicos na espécie felina (Pypendop et al., 2011; Rodrigo-Mocholi et al., 2016).

Vale salientar ainda que, em alguns momentos de estímulo supramáximo, foram ultrapassados os limites de referência (Camacho e Mucha, 2014) para PAS (M6) nos animais tratados com o tramadol e para PAS, PAM e PAD (M3 a M6) quando utilizada a dexmedetomidina. Tal evento possivelmente condiz com maior liberação de catecolaminas diante de estímulo doloroso, especialmente nas gatas que receberam o alfa-2 agonista, cujos efeitos vasoconstritores somamse ao estímulo adrenérgico já mencionado. 
Tabela 1. Valores médios $(\overline{\mathrm{x}})$ e desvio-padrão $( \pm \sigma)$ de FC (bpm), PAS, PAM e PAD $(\mathrm{mmHg}), f(\mathrm{mpm})$, $\mathrm{SpO}_{2}(\%), \mathrm{ETCO}_{2}(\mathrm{mmHg}), \mathrm{T}\left({ }^{\circ} \mathrm{C}\right)$ e taxas de infusão de propofol em gatas submetidas à anestesia epidural com lidocaína associada ao tramadol (GLT) ou à dexmedetomidina (GLD) e submetidas à ovariosalpingohisterectomia

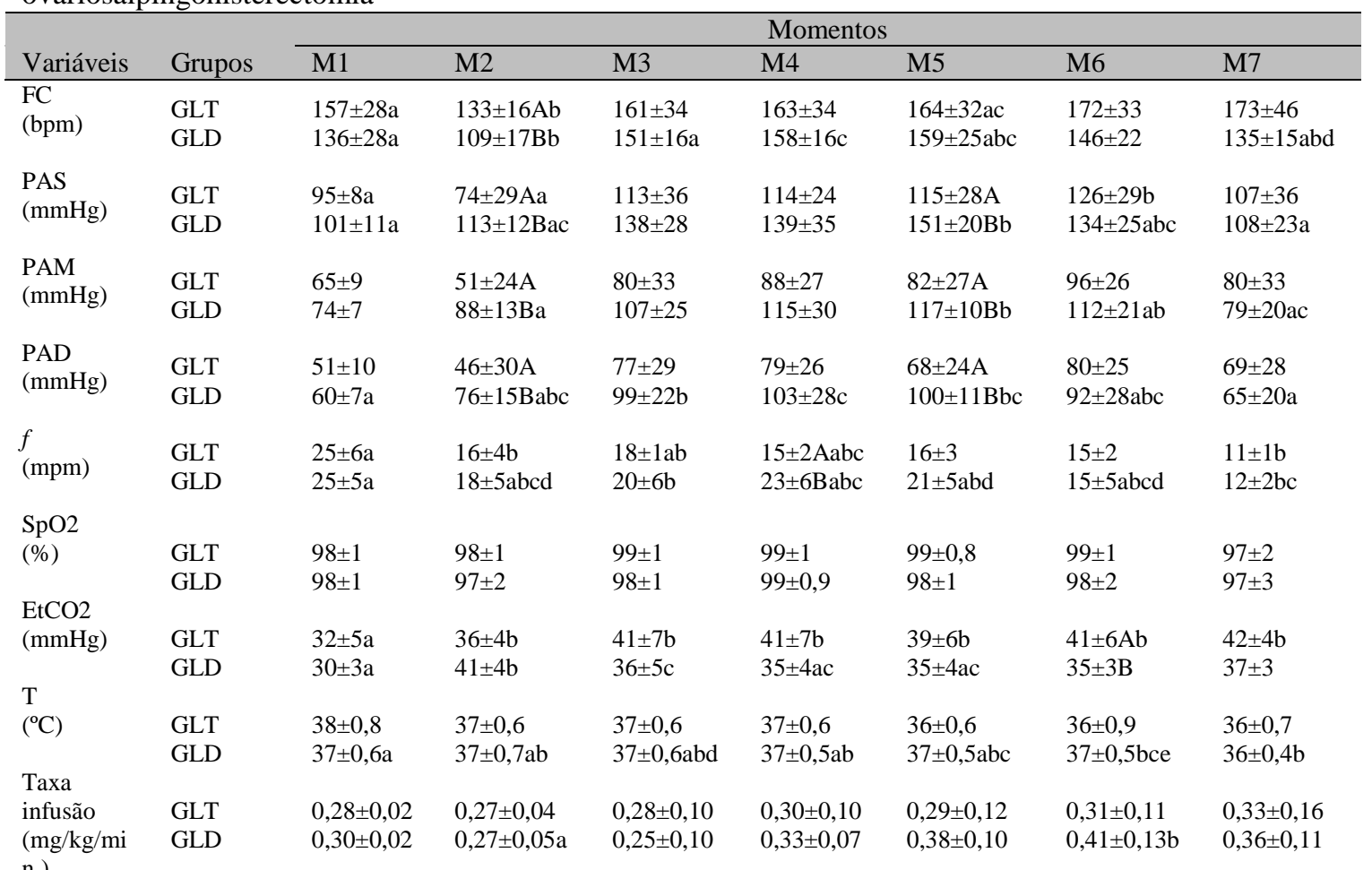

Médias seguidas por letras minúsculas diferentes, nas linhas, demonstram diferença estatística entre momentos $(\mathrm{P}<0,05)$. Médias seguidas por letras maiúsculas diferentes, nas colunas, demonstram diferença estatística entre grupos $(\mathrm{P}<0,05)$.

Relativamente à função respiratória (Tab. 1), constataram-se maiores médias no GLD em relação ao GLT no momento correspondente à ligadura do ovário direito (M4). Dessa forma, ratifica-se a assertiva de que os animais tratados com a dexmedetomidina estiveram mais sensíveis à liberação adrenérgica inerente ao estímulo doloroso, corroborando as variações pressóricas já discutidas. Ao longo do tempo, houve redução das médias iniciais para o GLT (M2 e M7) e para o GLD (M3 e M7), o que, de fato, pode ser imputado à infusão do alquifenol em felinos, visto que tanto o tramadol (Mastrocinque et al., 2012) quanto a dexmedetomidina (Dorigon et al., 2009; Lemos et al., 2017) são considerados seguros ao perfil respiratório.

A análise dos níveis de $\mathrm{EtCO}_{2}$ revelou médias significativamente menores (M6) para o GLD em relação ao GLT. De modo geral, exceto em M2, houve uma tendência a menores médias no grupo tratado com dexmedetomidina condizentes com os maiores valores de $f$, detectando-se, apenas no momento inicial, médias aquém do intervalo de normalidade $(35-45 \mathrm{mmHg})$, sem significativo clínico. No entanto, para ambos os protocolos, os achados de capnografia ratificam a ausência de depressão respiratória. Os níveis de $\mathrm{SpO}_{2}$ preservados enfatizam a estabilidade ora mencionada e permitem descartar episódios hipoxêmicos, independentemente do tratamento. A T $\left({ }^{\circ} \mathrm{C}\right)$ não diferiu entre os grupos, havendo sensível decréscimo ao longo do tempo. Apesar do uso do colchão térmico ativo, o miorrelaxamento acentuado, a vasodilatação e a depressão do centro termorregulador, em virtude da anestesia geral, podem implicar na perda de calor observada (Oliveira et al., 2007).

A taxa mínima de infusão de propofol não diferiu entre os tratamentos, evidenciando-se valores próximos ao inicial $(0,3 \mathrm{mg} / \mathrm{kg} / \mathrm{min})$, com taxas médias finais para o GLT e o GLD de 
$0,30 \pm 0,09 \mathrm{mg} / \mathrm{kg} / \mathrm{min}$ e $0,33 \pm 0,08 \mathrm{mg} / \mathrm{kg} / \mathrm{min}$., respectivamente. Dessa forma, pode-se aventar a eficácia analgésica dos fármacos testados, havendo a proximidade das médias com o limite inferior recomendado para manutenção anestésica $(0,3$ e $0,8 \mathrm{mg} / \mathrm{kg} / \mathrm{min}$ ) (Massone e Cortopassi, 2009), mesmo durante o estímulo álgico, sugerindo ações potencializadoras. A diminuição da taxa de infusão de anestésicos gerais intravenosos ou do consumo de halogenados é igualmente relatada por diferentes autores quanto ao uso epidural do tramadol, em razão de sua interação com receptores em toda extensão do canal espinhal (Oliveira Neta et al., 2014; Hermeto et al., 2015), bem como do uso da dexmedetomidina, pela mesma via, por proporcionar bloqueio sensitivo das fibras nervosas (Souza et al., 2010; Pohl et al., 2012; Caires e Clark, 2014). Apenas para o GLD, houve incremento da taxa de propofol ao final da sutura abdominal (M6) em relação ao período correspondente ao final da celiotomia (M2), fato que ratifica a maior sensibilidade álgica dos animais tratados com o alfa-2 agonista.

$\mathrm{Na}$ avaliação pós-operatória, não foram constatados efeitos adversos, como vômito, excitação e edema pulmonar, para ambos os protocolos. Contudo, os animais do GLD apresentaram recuperação mais prolongada e sedação mais pronunciada em comparação ao GLT (Tab. 2), de modo que algumas felinas tratadas com dexmedetomidina-lidocaína permaneceram profundamente sedadas por até 30 minutos após a cirurgia. Em estudo semelhante, Souza et al. (2010) relatam incremento do tempo de recuperação de gatas submetidas à OSH com o uso de dexmedetomidina epidural ou em infusão contínua, o que foi atribuído ao efeito sedativo do fármaco, independentemente da via de administração. Em contrapartida, Caires e Clark (2014) observaram maior duração de seu efeito sedativo (duas horas) pela via epidural, quando comparada à via sistêmica. De maneira oposta, não foram detectadas características sedativas com o uso do tramadol associado à lidocaína, em acordo com outras pesquisas (Monteiro et al., 2016; Castro et al., 2009). Complementarmente e de forma similar, a dexmedetomidina determinou maior efeito sedativo que o tramadol, quando combinados ao midazolam, em felinas submetidas à OSH (Lemos et al., 2017).

Tabela 2. Média $(\overline{\mathrm{x}})$ e desvio-padrão $( \pm \sigma)$ do tempo de recuperação anestésica de gatas submetidas à anestesia epidural com lidocaína associada à dexmedetomidina (GLD) ou ao tramadol (GLT) e submetidas à ovariosalpingohisterectomia

\begin{tabular}{ll}
\hline Grupos & Tempo (minutos) \\
\hline GLD & $24 \pm 12 \mathrm{~A}$ \\
GLT & $5 \pm 6 \mathrm{~B}$ \\
\hline
\end{tabular}

Médias seguidas por letras maiúsculas diferentes, nas colunas, demonstram diferença estatística entre grupos $(\mathrm{P}<0,05)$.

Durante a avaliação analgésica, não foram registradas diferenças significativas entre os escores no período pós-operatório (Tab. 3). Contudo, vale ressaltar que o resgate analgésico (escore de dor $\geq 8$ ) foi necessário no GLD em $25 \%$ (2), $25 \%$ (2) e $37,5 \%$ (3) dos animais nos momentos M60, M90 e M180, respectivamente, e em todas as gatas, considerando-se as seis horas de avaliação pós-cirúrgicas (Tab. 4). Em felinas submetidas à anestesia epidural com 0 mesmo alfa- 2 agonista $(2 \mu \mathrm{g} / \mathrm{kg})$ e igualmente anestesiadas com propofol e submetidas à OSH, a dexmedetomidina resultou em melhor analgesia e estabilidade anestésica em relação ao grupo placebo, contudo $33,3 \%$ dos animais apresentaram reação intensa pós-cirúrgica ao toque da ferida (Dorigon et al., 2009). Em adição, tanto a infusão intravenosa de dexmedetomidina quanto o uso epidural evidenciaram analgesia pós-operatória ineficaz em gatas sob o mesmo modelo cirúrgico (Souza et al., 2010). Por outro lado, a utilização do tramadol associado à lidocaína descartou resgate analgésico em $87,5 \%$ dos animais ao longo de todo o período avaliado (Tab. 4) em concordância com Castro et al. (2009), que relataram duração média de seis horas relativamente à analgesia proporcionada pelo tramadol epidural em felinos. Complementarmente, Lemos et al. (2017) evidenciaram resultados semelhantes, atribuindo menor efeito analgésico à dexmedetomidina, relativamente ao tramadol, quando associados ao midazolam, por via IM, em felinas. 
Avaliação da anestesia...

Tabela 3. Valores médios $(\overline{\mathbf{x}})$ e desvio-padrão $( \pm \sigma)$ do escore de dor, de acordo com escala multidimensional, de gatas submetidas à anestesia epidural com lidocaína associada à dexmedetomidina (GLD) ou ao tramadol (GLT) e submetidas à ovariosalpingohisterectomia

\begin{tabular}{llllllll} 
Grupos & Momentos & \multicolumn{7}{l}{} & & & \\
\hline & M30 & M60 & M90 & M120 & M180 & M240 & M360 \\
\hline GLD & $--^{*}$ & $13 \pm 6$ & $13 \pm 3$ & $11 \pm 4$ & $7 \pm 4$ & $6 \pm 4$ & $3 \pm 3$ \\
GLT & $3 \pm 2$ & $3 \pm 2$ & $4 \pm 3$ & $4 \pm 3$ & $3 \pm 3$ & $2 \pm 2$ & $2 \pm 2$ \\
\hline
\end{tabular}

*Animais profundamente sedados.

Tabela 4. Número de gatas que necessitaram de analgesia de resgate (escore de dor $\geq 8$ ), de acordo com escala multidimensional, tratadas com lidocaína associada à dexmedetomidina (GLD) ou ao tramadol (GLT), pela via epidural e submetidas à ovariosalpingohisterectomia

\begin{tabular}{lll} 
Grupos & $\begin{array}{l}\text { Gatas com } \\
\text { escore } \geq 8\end{array}$ & $\begin{array}{l}\text { Gatas com } \\
\text { escore }<8\end{array}$ \\
\hline GLD & 8 & 0 \\
GLT & 1 & 7 \\
\hline
\end{tabular}

\section{CONCLUSÕES}

Conclui-se que, quando associada à lidocaína, pela via epidural, a dexmedetomidina promove bradicardia inicial, elevação da pressão arterial sistêmica, maior tempo de recuperação e analgesia pós-operatória de menor qualidade, em comparação ao tramadol. Os protocolos promoveram efeitos similares sobre o consumo de propofol e asseguraram baixas taxas mínimas de infusão.

\section{REFERÊNCIAS}

BRONDANI, J.T.; LUNA, S.P.L.; MINTO, B.W. et al. Validade e responsividade de uma escala multidimensional para avaliação de dor pósoperatória em gatos. Arq. Bras. Med. Vet. Zootec., v.64, p.1529-1538, 2012.

CAIRES, L.; CLARK, R.M. Agonistas alfa-2 pela via epidural na analgesia de cães e gatos revisão de literatura. Vet. Zootec., v.21, p.359369,2014

CAMACHO, A.A.; MUCHA, C.J. Semiologia do sistema circulatório de cães e gatos. In: FEITOSA, F.L.F. Semiologia veterinária: a arte do diagnóstico. 4.ed. Roca, São Paulo, 2014. p.471-586.

CASTRO, D.; SILVA, M.F.; SHIH, A.C. et al. Comparison between the analgesic effects of morphine and tramadol delivered epidurally in cats receiving a standardized noxious stimulation. J. Feline Med. Surg., v.11, p.948953, 2009.
COMASSETTO, F.; GEHRCK, M.I.; LIMA, M.P.A. et al. Continuous infusion of propofol at variable rates in a time dependent in cats. Semin. Ciênc. Agrár., v.36, p.797-806, 2015.

DeROSSI, R.; BENITES, A.P.; FERREIRA, J.Z. et al. Effects of lumbosacral epidural ketamine and lidocaine in xylazine-sedated cats. J. S. Afr. Vet. Assoc., v.80, p.79-83, 2009.

DeROSSI, R.; HERMETO, L.C.; JARDIM, P.H.A. et al. Postoperative pain control in cats: clinical trials with pre-emptive lidocaine epidural co-administered with morphine or methadone. $J$. Feline Med. Surg., v.18, p.882-888, 2016

DORIGON, O.; OLESKOVICZ, N.; MORAES, A.N. et al. Dexmedetomidina epidural em gatas submetidas à ovariosalpingohisterectomia sob anestesia total intravenosa com propofol e prémedicadas com cetamina $\mathrm{S}(+)$ e midazolam. Cienc. Rural, v.39, p.791-797, 2009.

HERMETO, L.C.; DE ROSSI, R.; MARQUES, B.C. et al. Potentiation of epidural lidocaine by co-administering tramadol by either intramuscular or epidural route in cats. Can. J. Vet. Res., v.79, p.214-220, 2015.

KIM, D.S.; NA, H.S.; LEE, J.H. et al. Current clinical application of dexmedetomidine for sedation and anesthesia. Anesth. Pain Med., v.12, p.306-319, 2017.

KOCH, M.; DE BACKER, D.; VINCENT, J.L. et al. Effects of propofol on human microcirculation. Br. J. Anaesth., v.101, p.473478, 2008. 
KVOLIK, S.; SAKIC, K. The impacto f local anesthetics on perioperative on microcirculation and coagulation. J. Feline Med. Surg., v.109, p.283-287, 2007.

LEE, I.; YAMAGISHI, N.; OBOSHI, K. et al. Distribution of new methylene blue injected into the lumbosacral epidural space in cats. Vet. Anaesth. Analg., v.31, p.190-194, 2004.

LEMOS, V.C.C.; SANDE, J.Q.; BARBOSA, V.F. et al. Avaliação da dexmedetomidina e do tramadol, associados ao midazolam, em gatas anestesiadas com isoflurano e submetidas à ovário-histerectomia. Arq. Bras. Med. Vet. Zootec., v.69, p.1521-1528, 2017.

MASSONE, F.; CORTOPASSI, S.R.G. Anestesia Intravenosa. In: FANTONI, D.T.; CORTOPASSI, S.R.G. Anestesia em cães $e$ gatos. 2.ed. São Paulo: Roca, 2009. p.228-236.

MASTROCINQUE, S.; ALMEIDA, T.F.; TATARUNAS, A.C. et al. Comparison of epidural and systemic tramadol for analgesia following ovariohysterectomy. J. Am. Anim. Hosp. Assoc., v.48, p.310-319, 2012.

MONTEIRO, B.P.; KLINCK, M.P.; MOREAU, M. et al. Analgesic efficacy of an oral transmucosal spray formulation of meloxicam alone or in combination with tramadol in cats with naturally occurring osteoarthritis. Vet. Anaesth. Analg., v.43, p.643-651, 2016.

OLIVEIRA NETA, O.D.; OLESKOVICZ, N.; MORAES, A.N. Cardiopulmonary and analgesics effects of epidural morphine, fentanyl or tramadol in female dog undergoing a ovariohysterectomy. Rev. Bras. Cienc. Vet., v.36, p.281-288, 2014.

OLIVEIRA, F.A.; OLESKOVICZ, N.; MORAES, A.N. Anestesia total intravenosa em cães e gatos com propofol e suas associações. Rev. Ciênc. Agrovet., v.6, p.170-178, 2007.

OTERO, P.E. Anestesia Locorregional do Neuroeixo In: KLAUMANN, P.R.; OTERO, P.E. Anestesia locorregional em pequenos animais. São Paulo: Roca, 2013. p.135-176.
POHL, V.H.; CARREGARO, A.B.; LOPES, C. et al. Epidural anesthesia and postoperatory analgesia with alpha-2 adrenergic agonists and lidocaine for ovariohysterectomy in bitches. Can. J. Vet. Res., v.76, p.215-220, 2012.

PYPENDOP, B.H.; BARTER, L.; SCOTT, D.; ILKIW, J. Hemodynamic effect of dexmedetomidine in isoflurane-anesthetized cats. Vet. Anaesth. Analg., v.38, p.555-567, 2011.

RANKIN, D.C. Sedativos e tranquilizantes. In: GRIMM, K.A.; LAMONT, L.A.; TRANQUILLI, W.J.; GREENE, S.A.; ROBERTSON, S.A. Lumb and Jones anestesiologia e analgesia em veterinária. 5.ed. Rio de Janeiro: Roca, 2015. p.188-198.

RODRIGO-MOCHOLI, D.; BELDA, E.; BOSMANS, T. et al. Clinical efficacy and cardiorespiratory effects of intramuscular administration of alfaxalone alone or in combination with dexmedetomidine in cats. Vet. Anaesth. Analg., v.43, p.291-300, 2016.

SILVA, T.; CARVALHO, R.; TOLEDO, E.A. Dexmedetomidina um novo medicamento na anestesiologia veterinária. Rev. Cient. Eletrôn. Med. Vet., v.6, p.1-6, 2008.

SONAGLIO, F.; SILVA, J.P.; FERRANTI, O. Anestesia epidural em gatos. Rev. Agrocient., v.1, p.81-88, 2014.

SOUZA, S.S.; INTELISANO, T.R.; BIAGGI, C.P. et al. Cardiopulmonary and isoflurane-sparing effects of epidural or intravenous infusion of dexmedetomidine in cats undergoing surgery with epidural lidocaine. Vet. Anaesth. Analg., v.37, p.106-115, 2010.

TORSKE, K.E.; DYSON, D.H. End tidal halothane concentration and postoperative analgesia requirements in dogs: A comparison between intravenous oxymorphone and epidural bupivacaine alone and in combination with oxymorphone. Vet. Clin. N. Am. Small Anim. Pract., v.30, p.361-369, 2000.

VALVERDE, A. Epidural analgesia and anesthesia in dogs and cats. Vet. Clin. Small Anim., v.38, p.1205-1230, 2008. 